\title{
Theoretical analysis for sound-absorbing materials using layered narrow clearances between two planes
}

\author{
Shuichi SAKAMOTO*, Katsuya HIGUCHI**, Kazuyuki SAITO** and Shota KOSEKI** \\ * Department of Mechanical and Production Engineering, Niigata University \\ 2-8050 Ikarashi, Nishi-ku, Niigata 950-2181, Japan \\ E-mail: sakamoto@eng.niigata-u.ac.jp \\ ** Graduate School of Science and Technology, Niigata University \\ 2-8050 Ikarashi, Nishi-ku, Niigata 950-2181, Japan
}

Received 2 July 2014

\begin{abstract}
We investigated the sound absorption coefficient within a sound-absorbing structure made of layered clearances between two planes. We discussed the experimental results from the existing literature and conducted a theoretical analysis on several different sound-absorbing structures that take into account the viscosity of the boundary layer at the clearances. First, we examined the case of thin sheets placed in parallel. Then, we examined the case where the size of the clearance continuously changed perpendicular to the direction of incidence of the sound waves. Subsequently, we examined the case where the clearance continuously decreased in the direction of incidence of the sound waves. In latter two cases, the transfer matrix used in the calculations was divided into elements perpendicular or coincident to the direction of incidence of the sound waves and the size of the clearance for each element was changed in a stepwise fashion. We then calculated the sound absorption coefficients for various structures and dimensions and observed that the calculated and experimental data agreed well. On the basis of the calculation simulation, the differences between the calculated and experimental absorption coefficient data were attributed mainly to the changes in the flexure of the sheet materials. The proposed calculation methods will be useful in determining the absorption coefficient of such shapes, and in the design of sound-absorbing materials that use such shapes.
\end{abstract}

Key words : Sound and acoustics, Noise control, Porous media, Absorption coefficient, Theoretical analysis, Silencer design

\section{Introduction}

Porous bodies with continuous pores, exemplified by materials such as glass wool, are useful as sound-absorbing materials. The absorption coefficients of wheat straw (McGinnes, et al., 2005) and tatami (straw mat) (Tsuchida and Kobayashi, 1975) have been investigated as plant-derived, sound-absorbing structures. As an example of sound absorption using recycled paper, a low-frequency sound-absorbing body that used a Helmholtz silencer has been studied (Hirama, et al., 2003). One of the authors is studying the application of plant-derived structures with minute clearances as sound-absorbing materials (Sakamoto, et al. 2011).

This study aims to understand the absorption coefficient of sound-absorbing structures with layered clearances between two planes. We also report the experimental results for sound-absorbing structures in the existing literature (Sakamoto, et al., 2013a) Theoretical analyses which the viscosity of the boundary layer was taken into account have been carried out in the cylindrical tube (Tijdeman, 1975), and in the clearance between two parallel plates (Beltman,1998) (Stinson and Champou,1992). Consequently, the propagation constant could be derived. We also reported the sound-absorbing and transmission loss characteristics of cylindrical holes (Sakamoto, et al., 2013b) and thin louver slat silencer with build in Helmholtz resonators (Sakamoto, et al., 2014). In these cases, attenuation of the viscosity of the boundary layer was accounted.

In this study, we report the results of theoretical analyses on the absorption coefficients of various structures and dimensions with clearances between two planes constructed using sheet materials. First, we performed theoretical analysis of sound-absorbing structures using the clearance between two parallel planes. Then, for folding screen-type structures, we 
performed the analysis by dividing the transfer matrix in the direction perpendicular to the incident sound waves. Next, for wedge-shaped structures in which the clearance narrows in the direction in which the sound waves are incident, we split the transfer matrix in the direction of incident sound waves. In a theoretical analysis, the simulations were also carried out by varying two parameters: the nonuniformity in the thickness of the clearance and the flexure of the thin sheet material. We calculated the absorption coefficients of the objects that we analyzed and compared them with the measured absorption coefficients.

\section{Theoretical analysis}

\subsection{Theoretical analysis model}

We reported the experimental results (Sakamoto, et al., 2013a) for sound-absorbing structures that use of the friction due to the viscosity of the boundary layer. In the theoretical analyses, dimensions of analysis model Types A to C in the previous report (Sakamoto, et al., 2013a) were used. Then, the absorption coefficients of each sample were established theoretically and experimentally, following which they were compared. Table 1 lists the theoretical analyses model types and schematic diagrams. Table 2 lists the clearance of the thin sheets, the number of sheets, and the ratios of analyses model aperture. The third row in Table 1 is the incidence face, and the bottom row contains schematic views of the models from the side.

Table 1 Schematic drawings and dimension for theoretical analysis model

\begin{tabular}{|c|c|c|c|}
\hline Type & $\begin{array}{l}\text { Type A } \\
\text { Parallel }\end{array}$ & $\begin{array}{c}\text { Type B } \\
\text { Folding screen }\end{array}$ & $\begin{array}{l}\text { Type C } \\
\text { Wedge }\end{array}$ \\
\hline Clearance $d[\mathrm{~mm}]$ & $0.12,0.22,0.52$ & $0.22,0.51$ & $0.22,0.52$ \\
\hline $\begin{array}{c}\text { Incident face } \\
25.7 \times 25.7 \mathrm{~mm} \\
\text { Surrounded } 0.5 \mathrm{~mm} \text { casing }\end{array}$ & $\mid$\begin{tabular}{l||l}
$d$ \\
\end{tabular} & $d$ & \\
\hline $\begin{array}{l}\text { Side view } \\
\text { Length } \quad l=50 \mathrm{~mm}\end{array}$ & & & 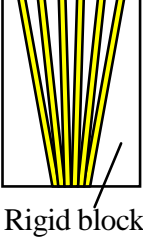 \\
\hline
\end{tabular}

Table 2 Typical specifications for theoretical analysis model

\begin{tabular}{ccccc}
\hline \multirow{2}{*}{ Type } & $\begin{array}{c}\text { Clearance on incident face } \\
{[\mathrm{mm}]}\end{array}$ & Number of thin sheets & Number of clearance & $\begin{array}{c}\text { Aperture ratio on } \\
\text { incident face }\end{array}$ \\
\hline \multirow{2}{*}{ Type A } & 0.12 & 124 & 123 & 0.553 \\
& 0.22 & 83 & 82 & 0.675 \\
\hline \multirow{2}{*}{ Type B } & 0.52 & 42 & 41 & 0.798 \\
& 0.22 & 83 & 82 & 0.675 \\
\hline \multirow{2}{*}{ Type C } & 0.51 & 43 & 42 & 0.795 \\
& 0.22 & 83 & 82 & 0.675 \\
\hline
\end{tabular}




\subsection{Four-terminal constants of unit acoustic elements}

The clearance between the two planes, which is based on the one-dimensional wave equation in the $x$-direction, is represented by the transfer matrix method that relates to sound pressure and volume velocity (Suyama and Hirata, 1979b). Fig. 1 shows the model of a sound wave incident in the positive $x$-direction inside a clearance of thickness $H$, which is created in a space with thickness $H_{0}$ in the $z$-direction.

$H_{0}$ is the unit thickness for each clearance including the non-opening section on test sample; i.e., the clearance thickness $H$ is divided by the aperture ratio. In Fig. 1, if the clearance thickness $H$ between two planes is sufficiently small compared to the length of the clearance in the $y$-direction, the propagation of the sound waves is determined by the $x$-and $z$-direction of the clearance, and is considered to be constant in the $y$-direction. For that reason, the cross-sectional area of the clearance in the $y-z$ plane can be replaced by $H$ : the product of the clearance thickness $H$ and the unit length in the $y$-direction. The transfer matrix $T$ and the four-terminal constants of unit acoustic elements $(A-D)$ are expressed with Eq. (1) that relates the length $l$, the characteristic impedance $Z_{\mathrm{c}}$, and the propagation constant $\gamma$.

$$
T=\left[\begin{array}{ll}
A & B \\
C & D
\end{array}\right]=\left[\begin{array}{cc}
\cosh (\gamma) & \frac{Z_{\mathrm{c}}}{H} \sinh (\gamma) \\
\frac{H}{Z_{\mathrm{c}}} \sinh (\gamma) & \cosh (\gamma)
\end{array}\right]
$$

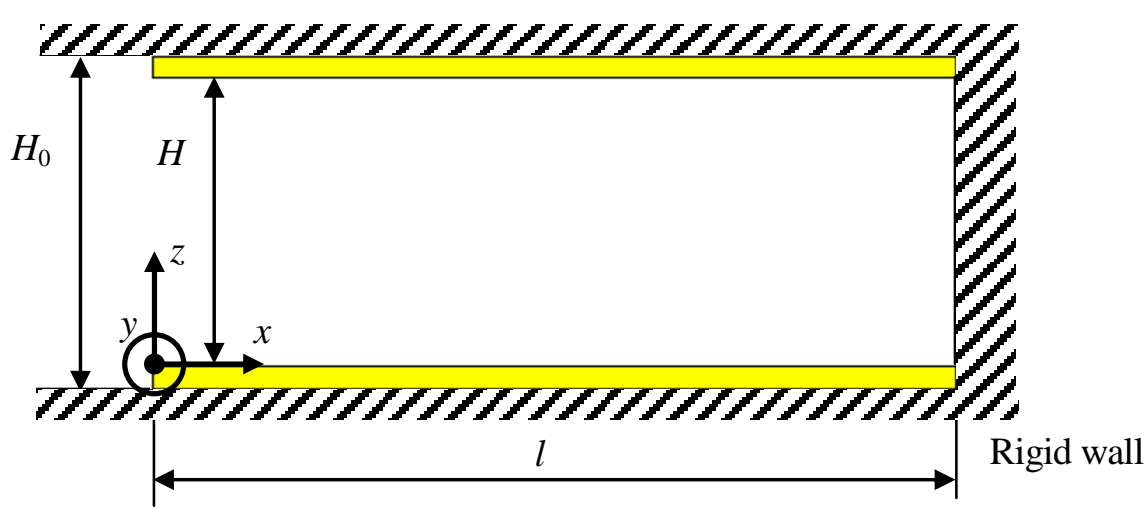

Fig. 1 Model of a narrow clearance between two planes

\subsection{The specific acoustic impedance $Z_{0}$ of the clearance between two parallel planes}

For sound pressures $p_{1}$ and $p_{2}$, and $x$ direction component of particle velocities $u_{1}$ and $u_{2}$ at the entrance and terminus of the clearance, respectively, the transfer matrix is expressed with Eq. (2). Because the terminus of the clearance is a rigid wall, the particle velocity $u_{2}=0$.

$$
\left[\begin{array}{c}
p_{1} \\
H u_{1}
\end{array}\right]=\left[\begin{array}{ll}
A & B \\
C & D
\end{array}\right]\left[\begin{array}{c}
p_{2} \\
H u_{2}
\end{array}\right]=\left[\begin{array}{c}
A p_{2} \\
C p_{2}
\end{array}\right]
$$

From the sound pressure $p_{0}$ and $x$ direction component of particle velocity $u_{0}$ in the upstream side in Fig. 1, the specific acoustic impedance $Z_{0}$ is expressed with left-hand side of Eq. (3) below. Furthermore, from Eqs. (2), $p_{0}=p_{1}$ and $H_{0} u_{0}=H u_{1}$, left-hand side of Eq. (3) can be transformed to right-hand side of Eq. (3). Consequently, it is noted that the specific acoustic impedance $Z_{0}$ increases because of the $H_{0}=H$ a reciprocal of the aperture ratio of the clearance.

$$
Z_{0}=\frac{p_{0}}{u_{0}}=\frac{p_{0}}{u_{0} H_{0}} H_{0}=\frac{p_{1}}{u_{1} H} H_{0}=\frac{\mathrm{A}}{\mathrm{C}} H_{0}
$$

Generally, the relationship between the specific acoustic impedance $Z_{0}$ and the reflectance $R$ at the incidence face is expressed by using Eq. (4), where $\rho_{\mathrm{s}}$ is the air density and $c$ is the speed of sound in air. 


$$
\frac{Z_{0}}{\rho_{\mathrm{s}} c}=\frac{1+R}{1-R}
$$

The theoretical value of the absorption coefficient is given by Eq. (5) below.

$$
\alpha=1-|R|^{2}
$$

\subsection{Propagation constant and characteristic impedance considering account attenuation of the sound waves in the clearance between the two planes}

For the attenuation of sound waves inside the tubes, the attenuation constant of tubes with internal diameters greater than $20 \mathrm{~mm}$ has been experimentally found (Suyama and Hirata, 1979a). Theoretical analyses which the viscosity of the boundary layer was taken into account have been carried out in the cylindrical tube (Tijdeman, 1975), and in the clearance between two parallel plates (Beltman,1998) (Stinson and Champou,1992). Consequently, the propagation constant could be derived. The thickness of the velocity boundary layer depends on the frequency (Nyborg, 1958), and it is estimated about $50 \mu \mathrm{m}$ at the 1 $\mathrm{kHz}$.

We have converted the cylindrical coordinate system of the Tijdeman method mentioned above into the Cartesian system to take into account the attenuation of the sound waves in the clearance between the two planes and have used the converted method.

Using approximations to the Navier-Stokes equation, the continuity equation, the equation of state for an ideal gas, and the energy equation in the $x$ - and $z$-directions of the Cartesian coordinate system in Fig. 1 , the $x$ direction component of particle velocity $u$ can be described with Eq. (6) (Tijdeman, 1975). In this case, the air was assumed to be a compressible fluid with constant viscosity. The assumed boundary conditions were such that the particle veloctiy in the $x$ - and $z$-directions on the wall surface of the clearance is zero, and the wall surface was assumed isothermal. In Eq. (6), $\kappa$ is the specific heat ratio, $s$ is the ratio of the clearance thickness and the velocity boundary layer thickness, $\sigma$ is the square root of the Prandtl number, and $\eta$ is the $z$ position in the $z$-direction normalized by the clearance thickness $H$. $\xi$ is the $x$ position in the $x$-direction expressed as $\xi=\omega x / c$ to make it dimensionless. Finally, $\omega$ is the angular frequency.

$$
j u=-\frac{1}{\kappa} \frac{\partial p}{\partial \xi}+\frac{1}{s^{2}} \frac{\partial^{2} u}{\partial \eta^{2}}
$$

For $u=f(\xi) h(a)$, Eq. (6) can be transformed to Eq. (7) with $a=j^{3 / 2} \eta s$.

$$
\frac{\partial^{2} h}{\partial a^{2}}+h=\frac{1}{\kappa f} \frac{\partial p}{\partial \xi}
$$

We assume that $h(a)=e^{j a}$ and a general solution to Eq. (7) can be obtained with Eq. (8), where $C_{1}, C_{2}$ are arbitrary constants.

$$
h(a)=C_{1} \mathrm{e}^{j a}+C_{2} \mathrm{e}^{-j a}+\frac{j}{\kappa f(\xi)} \frac{\partial p}{\partial \xi}
$$

Applying the boundary conditions to Eq. (8), the $x$ direction component of particle velocity $u$ can be expressed with Eq. (9).

$$
u=\frac{j}{\kappa} \frac{\partial p}{\partial \xi}\left[1-\frac{\sinh \left(j^{5 / 2} \eta s\right)-\sinh \left\{j^{5 / 2}(\eta-1) s\right\}}{\sinh \left(j^{5 / 2} s\right)}\right]
$$

Similarly, if we solve for temperature, and calculate for the $z$ direction component of particle velocity with the boundary condition applied, we obtain Eq. (10) that also includes the sound pressure $p$ (Tijdeman, 1975). 


$$
p\left[1-\frac{\kappa-1}{\kappa}\left\{1-2 \cdot \frac{\cosh \left(j^{\frac{5}{2}} \sigma s\right)-1}{j^{\frac{5}{2}} \sigma \sinh \left(j^{\frac{5}{2}} \sigma s\right)}\right\}+\frac{1}{\kappa} \frac{\partial^{2} p}{\partial \xi^{2}}\left\{1-2 \cdot \frac{\cosh \left(j^{\frac{5}{2}} s\right)-1}{j^{\frac{5}{2}} s \sinh \left(j^{\frac{5}{2}} s\right)}\right\}=0\right.
$$

Furthermore, if we replace the sound pressure $p$ with Eq. (11), the propagation constant $\gamma$ for the clearance between the two planes is given by Eq. (12) (Tijdeman, 1975), where $X, Y$ are arbitrary constants and $k$ is a wave number.

$$
\begin{gathered}
p=X \mathrm{e}^{\gamma x}+Y \mathrm{e}^{-\gamma x} \\
\gamma=k \sqrt{\frac{\cosh \left(j^{\frac{5}{2}} s\right)-1}{2 \cdot \frac{5}{j^{\frac{5}{2}} s \sinh \left(j^{\frac{5}{2}} s\right)}-1}}-1 \quad \sqrt{\frac{\kappa}{N}} \quad N=\left[1+\frac{\kappa-1}{\kappa}\left\{2 \cdot \frac{\cosh \left(j^{\frac{5}{2}} \sigma s\right)-1}{j^{\frac{5}{2}} \sigma s \sinh \left(j^{\frac{5}{2}} \sigma s\right)}-1\right\}\right]^{-1}
\end{gathered}
$$

Next, we discuss the characteristic impedance $Z_{c}$ for the clearance between the two planes, taking into account the attenuation.

If the $x$ direction component of particle velocity and sound pressure of the travelling wave are $u^{+}$and $p^{+}$, respectively, the characteristic impedance $Z_{\mathrm{c}}$ is expressed with Eq. (13).

$$
Z_{\mathrm{c}}=\frac{p^{+}}{u^{+}}
$$

The $u^{+}$and $p^{+}$inside the clearance between the two planes with attenuation are given by Eqs. (14) and (15), respectively (Tijdeman, 1975). $P_{\mathrm{s}}$ is the atmospheric pressure and $t$ is time.

$$
\begin{gathered}
u^{+}=c \frac{j \gamma}{\kappa k}\left[1-\frac{\sinh \left(j^{5 / 2} \eta s\right)-\sinh \left\{j^{5 / 2}(\eta-1) s\right\}}{\sinh \left(j^{5 / 2} s\right)}\right]\left(-Y \mathrm{e}^{-\chi x}\right) \mathrm{e}^{j \omega t} \\
p^{+}=P_{\mathrm{s}} Y \mathrm{e}^{-2 x} \mathrm{e}^{j \omega t}
\end{gathered}
$$

The characteristic impedance $Z_{\mathrm{c}}$ obtained is a function of the $z$-direction of the clearance. To make $Z_{\mathrm{c}}$ a function of the $x$-direction only, we average $Z_{\mathrm{c}}$ by integrating with respect to $z$-direction over the $y-z$ cross-section and $\overline{Z_{\mathrm{c}}}$, which is uniform on the $y-z$ cross-section, is estimated with Eq. (16).

$$
\overline{Z_{\mathrm{c}}}=\frac{1}{H} \int_{0}^{H} Z_{\mathrm{c}} d z
$$

In the above discussion, the propagation constant $\gamma$ and the characteristic impedance $\overline{Z_{\mathrm{c}}}$ of the clearance between the two planes were estimated while taking into account the attenuation. By substituting for $\gamma$ and $\overline{Z_{\mathrm{c}}}$ in Eq. (2), we can introduce the two-dimensional results for the clearance between the two planes to the one-dimensional transfer matrix.

The calculated results of propagation constant $\gamma$ and characteristic impedance $\bar{Z}_{\mathrm{c}}$ for the various clearance thicknesses are shown in Figs. 2-5. Now, the real and imaginary parts of the propagation constant were converted to attenuation constant and phase velocity, respectively. The real and imaginary parts of the characteristic impedance were normalized by that in air. 
The calculated results using Stinson (Stinson and Champou,1992) and Allard (Allard and Atalla, 2009) methods are also shown in same figures. The calculated results using Stinson and Allard method and those using author's method are close.

By comparing with the case of the larger clearance (Sakamoto, et al., 2014), attenuation constant and magnitude of characteristic impedance are greater, and phase velocity is smaller. These trends of Figs. 2-5 are similar to those of porous materials in which solid-borne sound can be ignored (Koshiroi and Tateishi, 2012).

By the way, to measuring the experimental value of propagation constant and characteristic impedance of test sample, there is the two cavity method (Utsuno, et al., 1989). However, this method is unusable because the terminal ends of the test samples constructed in our study are sealed.

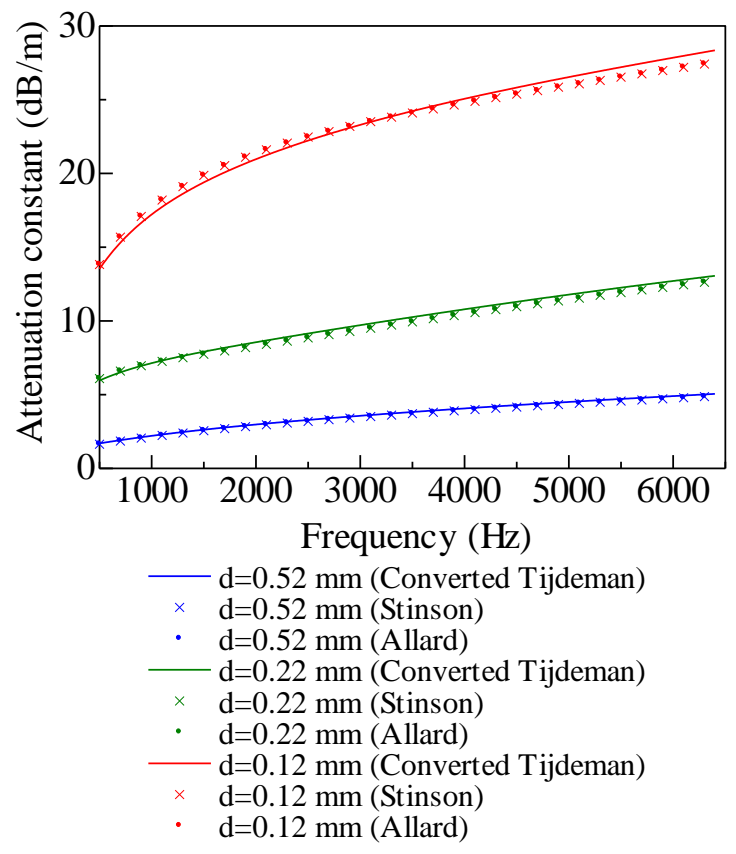

Fig. 2 Attenuation constant of $\mathrm{dB} /$ meter

(Type A, $20 \log _{10} \exp (\operatorname{Re}(\gamma))=8.6859 \operatorname{Re}(\gamma)$ )

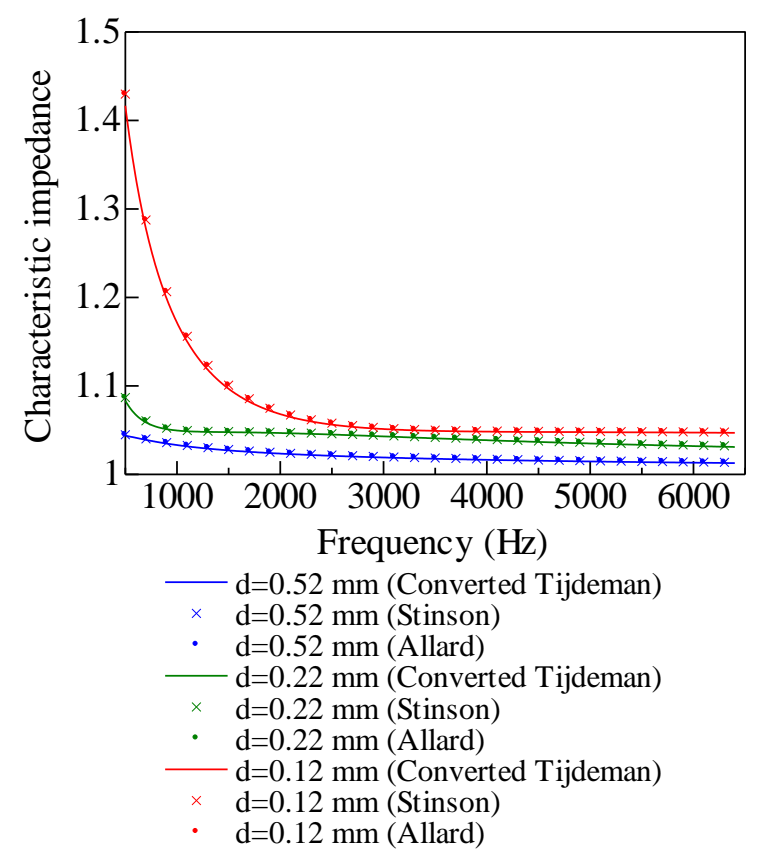

Fig. 4 Characteristic impedance $\operatorname{Re}\left(\overline{Z_{\mathrm{c}}}\right)$ by Eq. (16)

(Type A, Normalized by $Z_{\text {air }}$ )

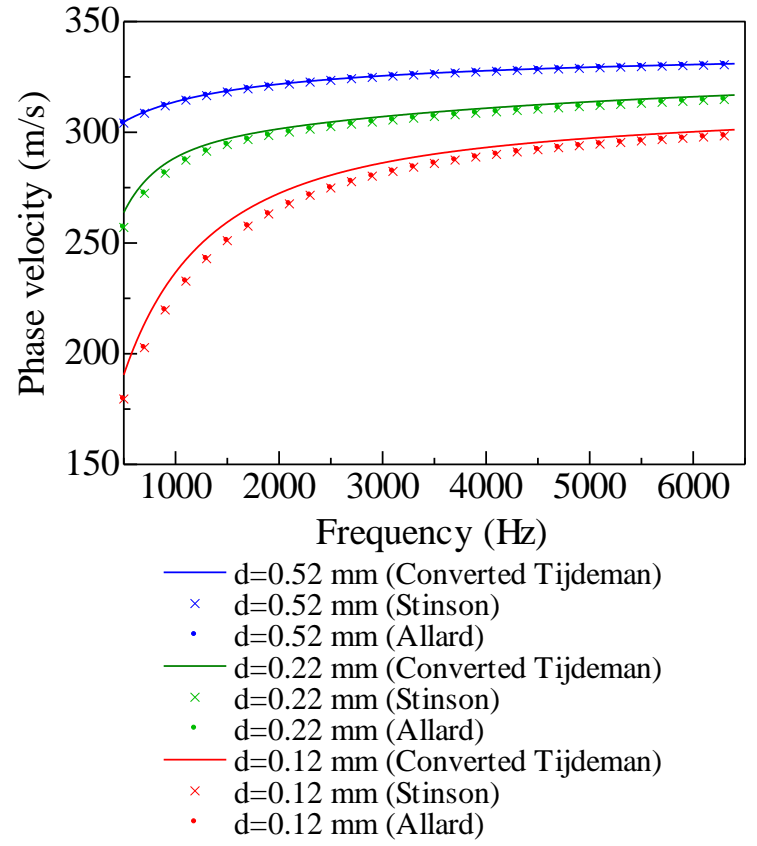

Fig. 3 Phase velocity

(Type A, $\omega / \operatorname{Im}(\gamma)$ )

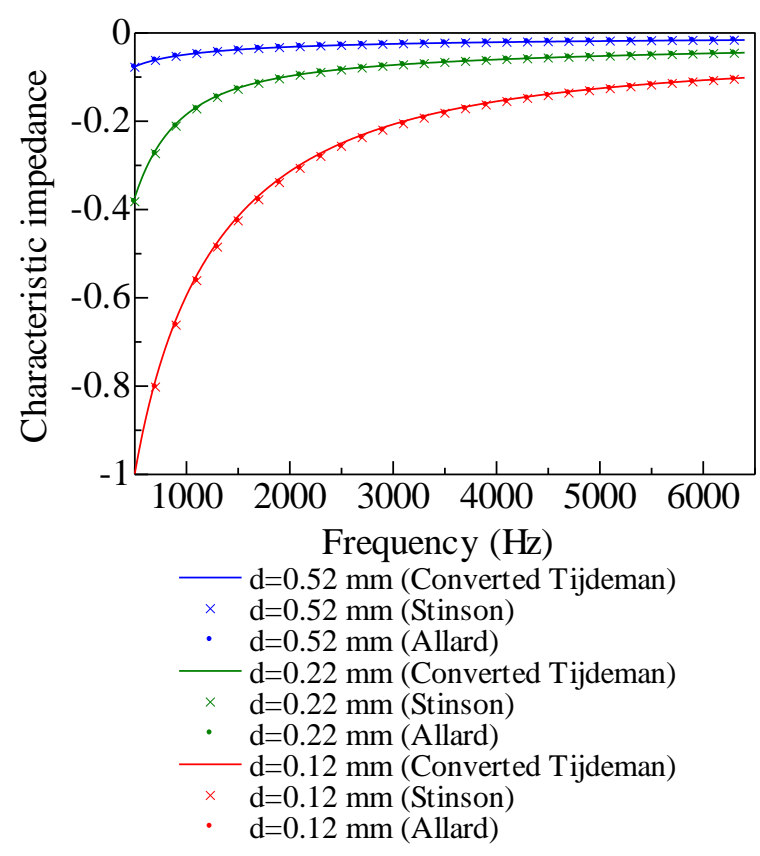

Fig. 5 Characteristic impedance $\operatorname{Im}\left(\overline{Z_{\mathrm{c}}}\right)$ by Eq. (16)

(Type A, Normalized by $Z_{\text {air }}$ ) 


\subsection{Derivation of the transfer matrix for Type $B$}

To derive the specific acoustic impedance of the clearance for the Type B shape, the clearance of the wedge-shaped cross-section in the $y-z$ plane in Fig. 6(a) is approximated as in Fig. 6(b). In other words, the clearance of thickness $H_{\mathrm{b}}$ is treated as $n$ clearances consisting of pairs of parallel planes that change in $n$ steps.

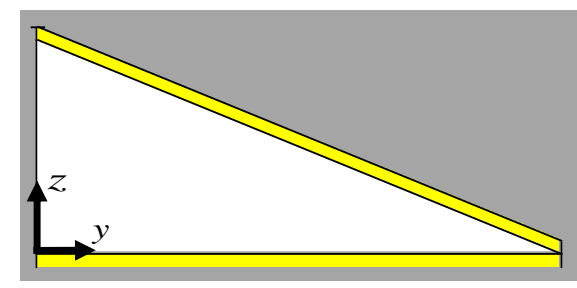

(a) Actual

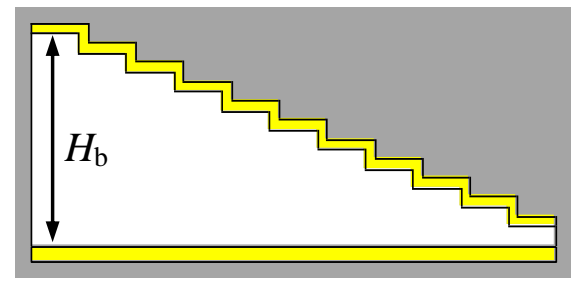

(b) Approximated

Fig. 6 Approximation of clearance shape on Type B

It therefore follows that the Type B transfer matrix can be expressed as the equivalent circuit in Fig. 7 by the parallel connection of the transfer matrices for each $n$ clearance of the parallel pairs of planes. In this case, each element is considered that the independent clearance, so that propagation of the sound pressure and particle velocity between elements is ignored (Verdière, 2013).

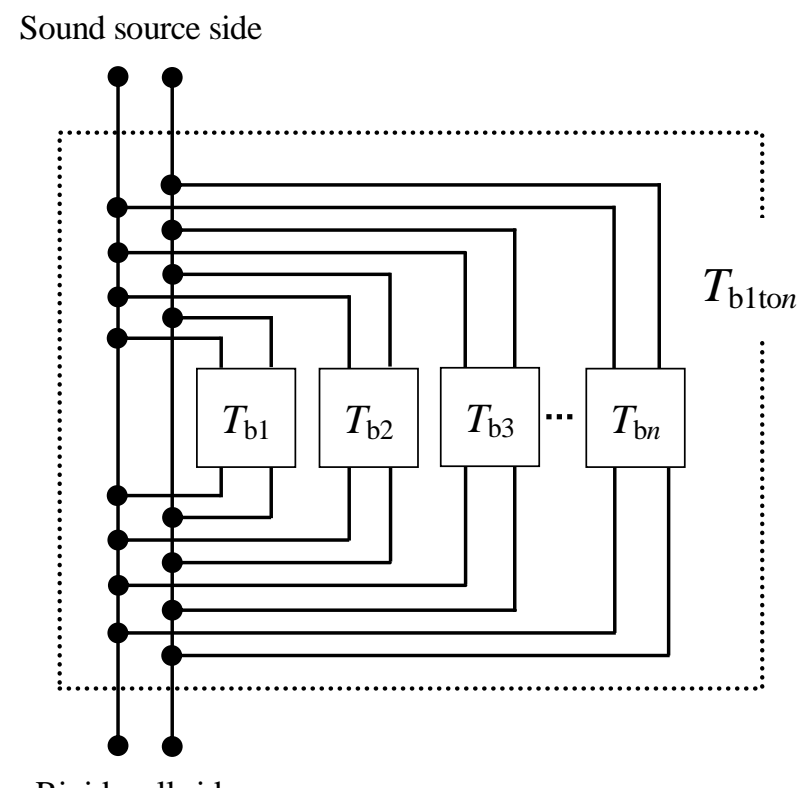

Rigid wall side

Fig. 7 Equivalent circuit for each clearance of Type B

If we add the transfer matrices for the "clearances connected in parallel" $T_{\mathrm{b} 1 \text { ton-1 }}$ and the "next clearance to be added" $T_{\mathrm{b} n}$ in Eq. (17), the transfer matrix for the connected in parallel $T_{\mathrm{blton}}$ can be expressed as with Eq. (18).

$$
T_{\mathrm{b} n}=\left[\begin{array}{ll}
A_{\mathrm{b} n} & B_{\mathrm{b} n} \\
C_{\mathrm{b} n} & D_{\mathrm{b} n}
\end{array}\right] \quad T_{\mathrm{b} 1 \mathrm{ton}-1}=\left[\begin{array}{ll}
A_{\mathrm{b} 1 \mathrm{ton}-1} & B_{\mathrm{b} 1 \mathrm{ton} n-1} \\
C_{\mathrm{b} 1 \mathrm{ton}-1} & D_{\mathrm{b} 1 \mathrm{ton}-1}
\end{array}\right]
$$




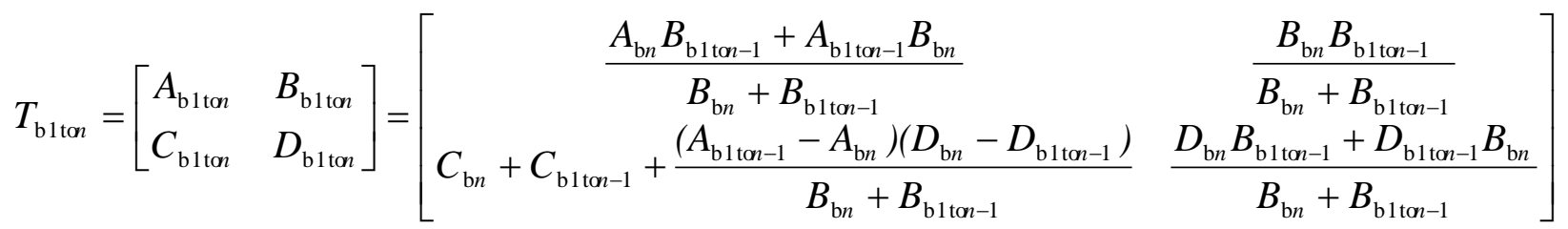

Replacing Eq. (2) with Eq. (18) for the transfer matrix $T_{\text {blton }}$, the specific acoustic impedance and absorption coefficient for each Type B clearance are estimated. The four-terminal constants $A_{\mathrm{b} n}-D_{\mathrm{b} n}$ in $T_{\mathrm{b} n}$ are given by using Eq. (2). $H$ in Eq. (2) is replaced by $H_{\mathrm{b}}$ that progressively decreases stepwise in the $y$-direction .

\subsection{Derivation of the transfer matrix for Type $C$}

To derive the specific acoustic impedance of the clearance for the Type $\mathrm{C}$ shape, the clearance of the wedge-shaped cross-section in the $x-z$ plane in Fig. 8(a) is approximated as in Fig. 8(b). In other words, the clearance of thickness $H_{\mathrm{c}}$ is treated as a clearance between two parallel planes that change in $i$ steps in the $x$-direction.

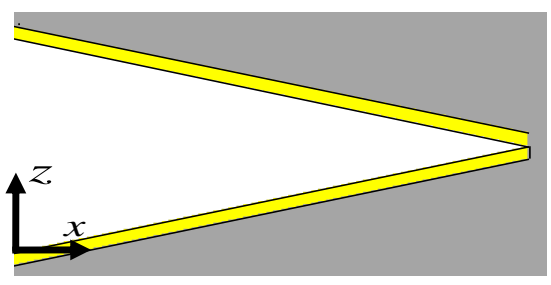

(a) Actual

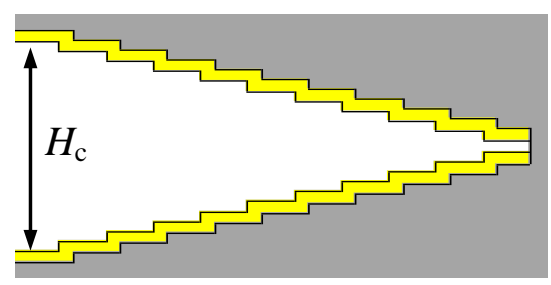

(b) Approximated

Fig. 8 Approximation of clearance shape on Type C

It follows that the transfer matrix for each clearance in Type $\mathrm{C}$ can be represented by an equivalent circuit as in Fig. 9, which is a cascade connection of the transfer matrices for the clearances between $i$ pairs of two parallel planes.

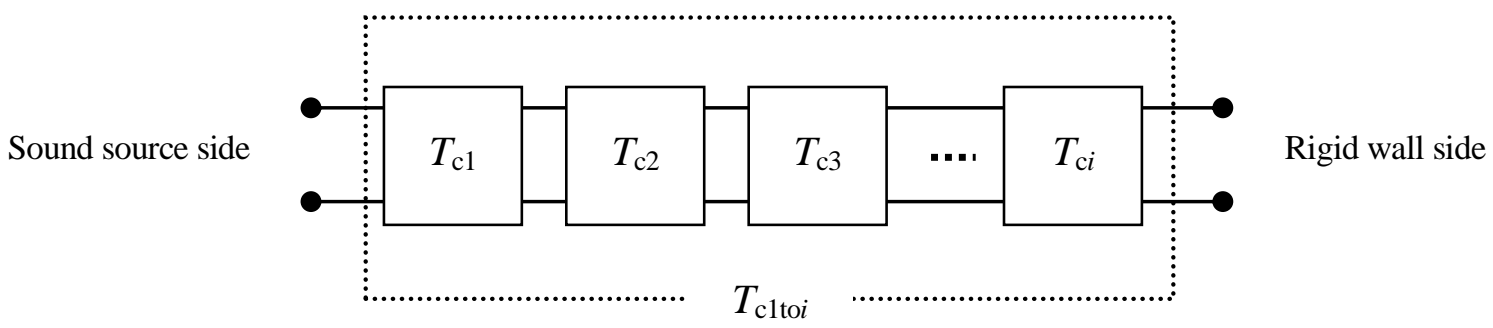

Fig. 9 Equivalent circuit for each clearance of Type C

Expressing the equivalent circuit in Fig. 9 with the transfer matrix, we obtain Eq. (19). The four-terminal constants $A_{\mathrm{c} i}-D_{\mathrm{c} i}$ for $T_{\mathrm{c} i}$ are given by Eq. (2). In this case, the length of clearance in the $x$-direction $l$ in Eq. (2) can be replaced by $l / i$, where $i$ is the number of divisions. Similarly, $H$ in Eq. (2) progressively decreases stepwise in the $x$-direction.

$$
T_{\mathrm{c} 1 \text { toi }}=\left[\begin{array}{ll}
A_{\mathrm{c} 1+0 i} & B_{\mathrm{c} 1 \mathrm{toi}} \\
C_{\mathrm{c} 10 i} & D_{\mathrm{c} 10 i}
\end{array}\right]=\left[\begin{array}{ll}
A_{\mathrm{c} 1} & B_{\mathrm{c} 1} \\
C_{\mathrm{c} 1} & D_{\mathrm{c} 1}
\end{array}\right]\left[\begin{array}{ll}
A_{\mathrm{c} 2} & B_{\mathrm{c} 2} \\
C_{\mathrm{c} 2} & D_{\mathrm{c} 2}
\end{array}\right] \ldots\left[\begin{array}{ll}
A_{\mathrm{c} i} & B_{\mathrm{c} i} \\
C_{\mathrm{c} i} & D_{\mathrm{c} i}
\end{array}\right]
$$

Replacing Eq. (2) with Eq. (19) for the transfer matrix $T_{\text {cltoi }}$, the specific acoustic impedance and adsorption coefficient for the each Type $\mathrm{C}$ clearance are estimated. 


\subsection{Derivation of the specific acoustic impedance for the whole sample}

Up to this point, individual clearance has been analyzed to find the specific acoustic impedance. Because there are multiple clearances for the samples used in the experiments, the specific acoustic impedances for each of the clearances are connected in parallel to represent the whole sample. If we assume specific acoustic impedances for the $m$ clearances to be $Z_{01}-Z_{0 m}$, we obtain the equivalent circuit for the $m$ parallel clearances as in Fig. 10 . In this case, the specific acoustic impedance for the whole sample is expressed with Eq. (20). For the case when all clearances have the same shape, all $Z_{01}-Z_{0 m}$ are equal.

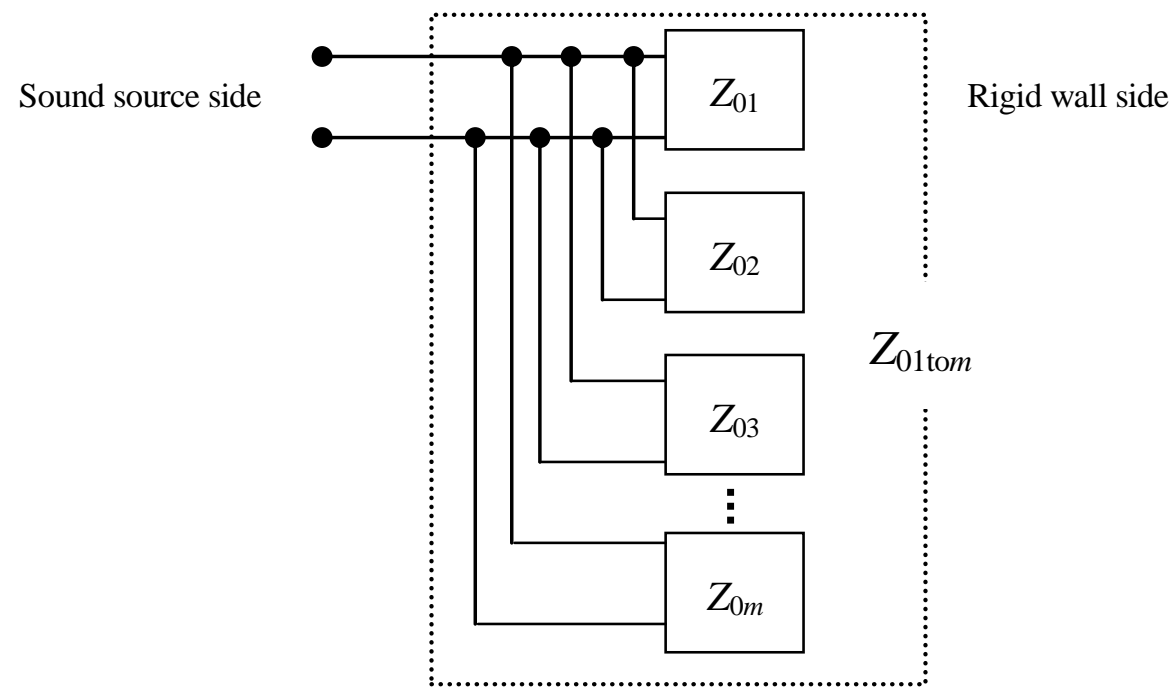

Fig. 10 Equivalent circuit for all clearances

$$
Z_{01 \mathrm{tom}}=\frac{1}{\frac{1}{Z_{01}}+\frac{1}{Z_{02}}+\frac{1}{Z_{03}}+\cdots+\frac{1}{Z_{0 m}}}
$$

Substituting $Z_{01 \text { tom }}$ in Eq. (20) with $Z_{0}$ in Eq. (4), the absorption coefficient for the whole sample can be estimated.

\section{Comparison of calculated and measured data \\ 3.1 Type A case}

Wood free paper (thickness $0.08 \mathrm{~mm}$ ) was used in the previous report (Sakamoto, et al. 2013a) for the materials constituting the clearances; however, in this study, we used stainless steel thin sheets (thickness $0.08 \mathrm{~mm}$ ). The change in the sound-absorbing characteristics achieved using the thin sheet material is explained in Section 3.4. The normal incident absorption coefficient was computed according to the ISO 10534-2 standard test method using the measured transfer function.

The calculated and measured absorption coefficient data are compared in Figs. 11-13 for each of the clearance thicknesses for the Type A sample. The calculated results using Stinson (Stinson and Champou,1992) and Allard (Allard and Atalla, 2009) method are also presented in Fig. 11. Also, the calculated absorption coefficients for the various clearance thicknesses are shown in Fig. 14, whereas the measured absorption coefficients are shown in Fig. 15. For any clearance thickness, the calculated and measured values exhibit a similar trend, which demonstrates the validity of the theoretical analysis. In particular, the calculated and measured absorption coefficients for an clearance thickness of $0.22 \mathrm{~mm}$ were extremely close. The calculated results using Stinson and Allard method and those using author's method are close.

For clearance thicknesses of 0.52 and $0.22 \mathrm{~mm}$, and any frequency, the calculated absorption coefficients were lower than the measured ones. The reasons for this discrepancy are probably in the approximation used in the theoretical analysis, and the assumptions that the size of the clearances is uniform and that the wall surface is flat. We consider the effect of nonuniformity of the clearances on the absorption coefficient in Section 3.4.

Additionally, in the case of a $0.12 \mathrm{~mm}$ thick clearance as in Fig. 13, the calculated absorption coefficients over a wide 
range of frequencies were higher than the experimentally established coefficients. Thus, we infer the following. From the calculated values in Fig. 14, we see that the peak value of the absorption coefficient increases as the clearance thickness decreases, reaching a maximum value at $0.16 \mathrm{~mm}$. As the clearance thickness decreases, the dip in the absorption coefficient also decreases; nonetheless, the absorption coefficient decreased over a wide range of the frequency. We also see from Fig. 15 that the experimental values exhibit a similar trend as the calculated values in Fig. 14.

We focus on the calculated values in Fig. 14 that correspond to the three conditions for the experimental results in Fig. 15. When the clearance thickness gradually decreases, compared to the experimental curve, the movement of the calculated curve is slightly delayed. In other words, if the clearance thickness in the calculations is slightly smaller than the experimental clearance, the agreement is better. This indicates that the calculated values for the attenuation of the sound waves are smaller than the actual values.

Moreover, in every test sample, frequency of the peak of calculated sound absorbing coefficient is higher than that of experimental one. The reason for this is that the calculated value for reduction of phase velocity in the clearance is lower than the actual one because the calculated value for attenuation of sound is also lower than the actual value.

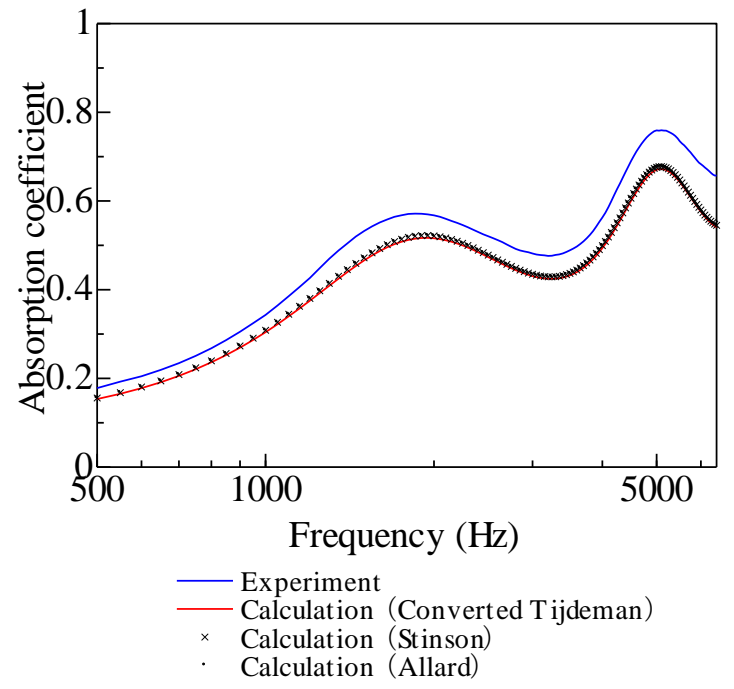

Fig. 11 Comparison between calculation and experiment

(Type A, $d=0.52 \mathrm{~mm}$ )

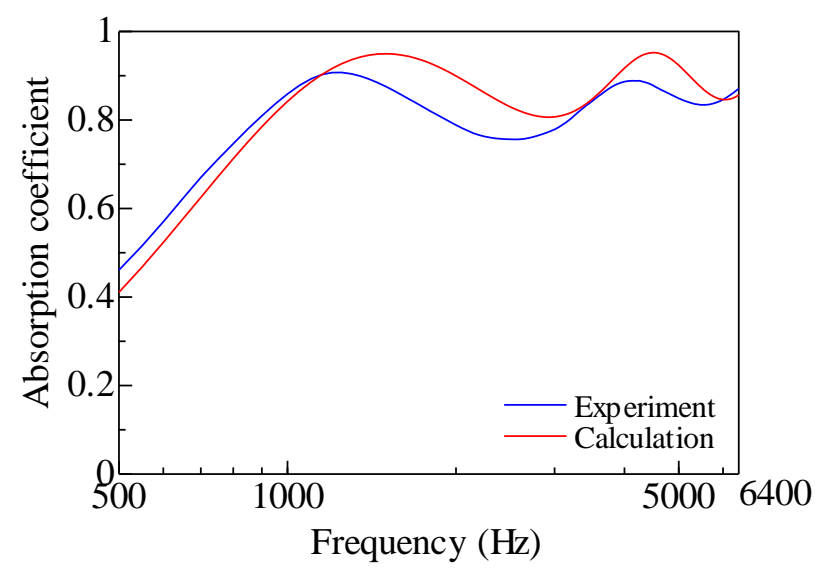

Fig. 13 Comparison between calculation and experiment

(Type A, $d=0.12 \mathrm{~mm}$ )

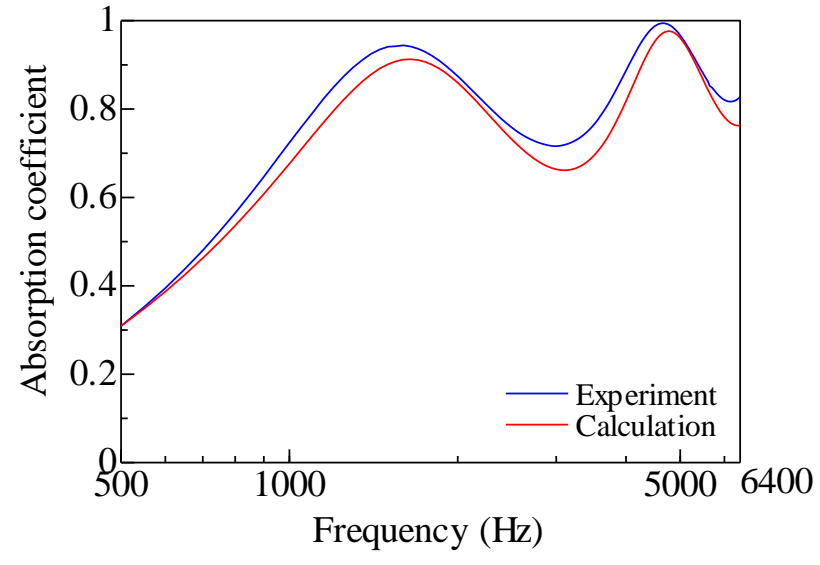

Fig. 12 Comparison between calculation and experiment

(Type A, $d=0.22 \mathrm{~mm}$ ) 

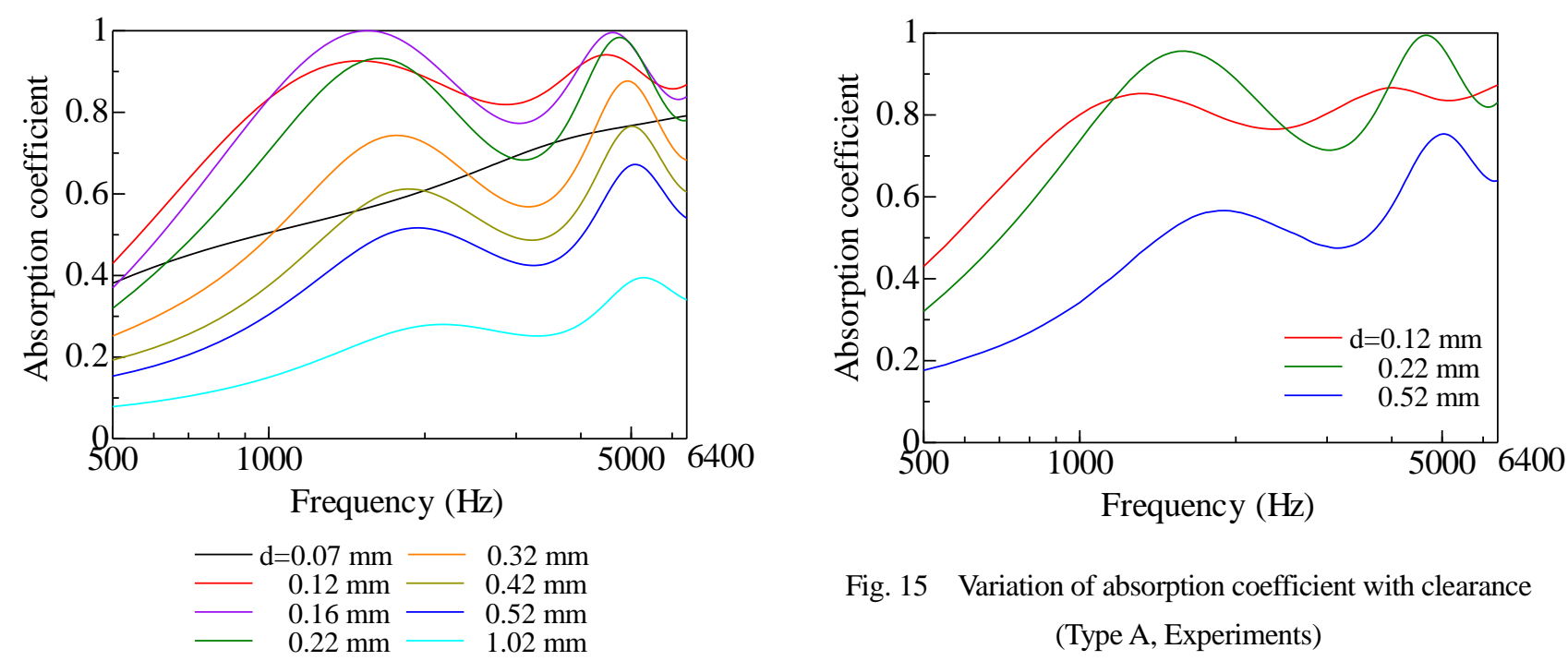

Fig. 15 Variation of absorption coefficient with clearance (Type A, Experiments)

Fig. 14 Variation of absorption coefficient with clearance

(Type A, Calculations)

\subsection{Type B case}

We compared the calculated and measured absorption coefficients with respect to the clearance thickness variations in Type B samples. The results are shown in Figs. 16 and 17. The number of division $n$ for Type B calculations was 50. This is a number where the calculated values are sufficiently converged for Type B. For comparison, the Type A results are also presented. The Type A and Type B experimental results are in agreement as shown in the previous report (Sakamoto, et al., 2013a). Similar trends are also seen in the calculated results for both Type A and Type B. Because the clearance thickness for Type B varies in the $y$-direction (see Fig. 6), the sound-absorbing capability varies depending on the position in the $y$-direction. However, by aligning the divided elements in parallel as in Fig. 7, the different absorption characteristics of the individual elements average out, and the end result becomes similar to Type A.

The calculated and measured values match closely, as in Type A. It shows that the theoretical analysis approximation by dividing the wedge-shaped clearance in the $y-z$ plane, as clearances between two planes, is an effective method. On the other hand, the calculated values were lower than the experimental ones. And frequency of the peak of calculated sound absorbing coefficient is higher than that of experimental one. These reasons for such are likely the same as in Type A, as discussed in Section 3.1 .

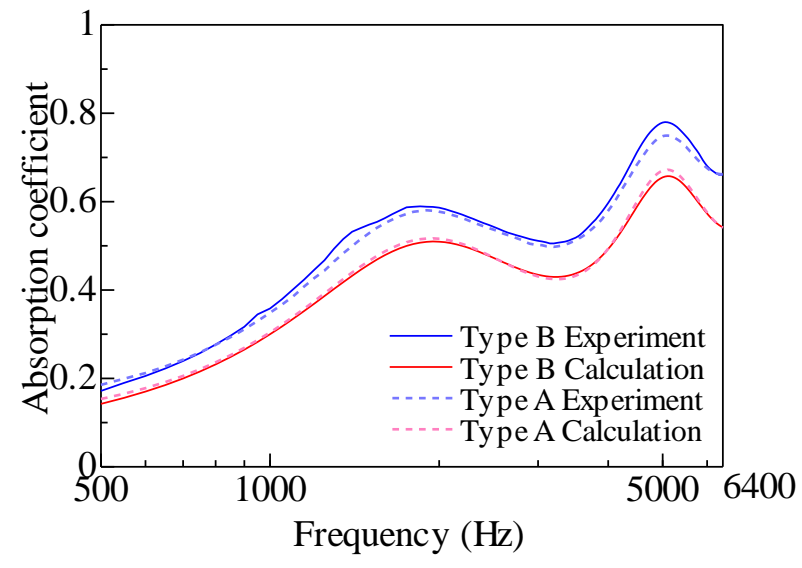

Fig. 16 Comparison between calculation and experiment

(Type A, $d=0.52 \mathrm{~mm}$ and Type $\mathrm{B}, d=0.51 \mathrm{~mm}$ )

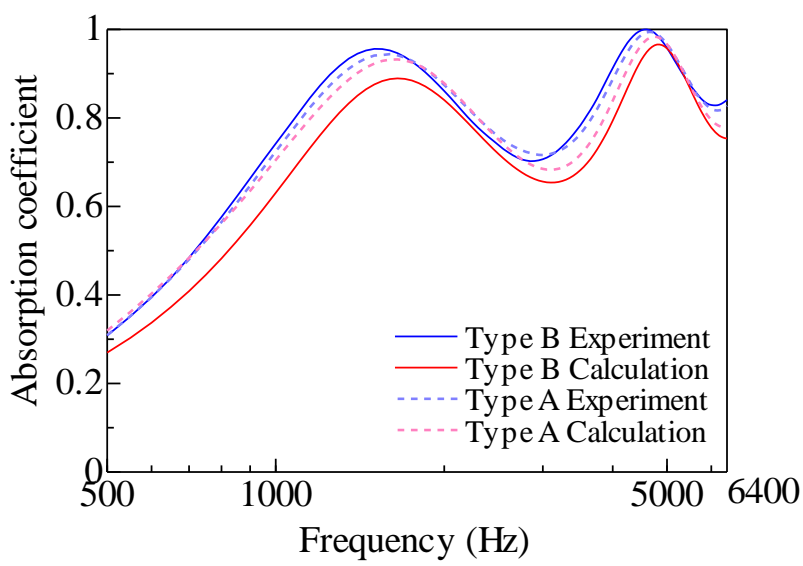

Fig. 17 Comparison between calculation and experiment (Type A, $d=0.22 \mathrm{~mm}$ and Type $\mathrm{B}, d=0.22 \mathrm{~mm}$ ) 


\subsection{Type $C$ case}

We compared the calculated and experimental absorption coefficients when the clearance thickness in Type C samples was varying. The results are shown in Figs. 18 and 19. The number of divisions $i$ in the Type $\mathrm{C}$ calculations was 100 . This is a number where the calculated values are sufficiently converged for Type C. For comparison, they are shown together with the Type A results. The trend in the calculated values closely follows the experimental values similar to the comparisons made above. This suggests the effectiveness of the theoretical analysis, which approximates the wedge shape of the clearance in the $x-z$ plane as clearances between two planes.

Compared with Type A, the frequency characteristics of the absorption coefficients in Type C samples shift toward the high-frequency side. The reason for this, as explained in the previous literature (Sakamoto, et al., 2013a), is that in Type C samples the clearance approaches 0 near the end of the $x$-direction, which means that the sound waves do not propagate to the end, and the effective length of the sample is shorter than in Type A samples. In the Type C samples, the clearance continuously decreases in the $x$-direction; therefore, the proportion of the boundary layer that occupies the space up to the terminal end increases, and the sound-absorbing capability over the wide frequency range improves. Hence, the dip in the curve of the absorption coefficient is improved.

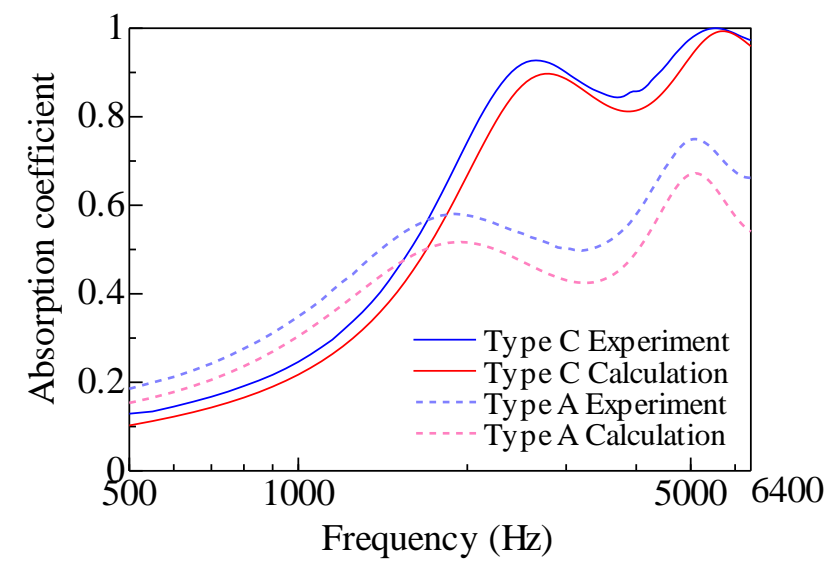

Fig. 18 Comparison between calculation and experiment

(Type A, $d=0.52 \mathrm{~mm}$ and Type $\mathrm{C}, d=0.52 \mathrm{~mm}$ )

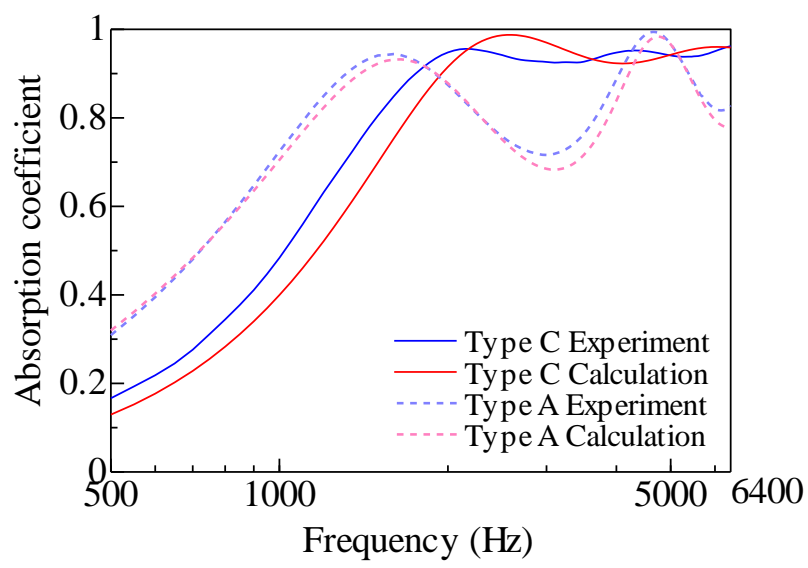

Fig. 19 Comparison between calculation and experiment

(Type A, $d=0.22 \mathrm{~mm}$ and Type $\mathrm{C}, d=0.22 \mathrm{~mm}$ )

\subsection{The effect of the clearance nonuniformity on the absorption coefficient}

In the theoretical analysis of the preceding sections, the position of the thin sheet was for samples with no offset, or flexure, and thus the clearance thickness was assumed uniform. However, the nonuniformity of the clearance may in fact affect the absorption coefficient. In the experiments, a stainless steel sheet of thickness $0.08 \mathrm{~mm}$ was used as the thin sheet material in order to exclude the effect of nonuniformity in the clearance. For comparison, we discuss the experimental values when using a paper (Sakamoto, et al., 2013a) with the same thickness as the stainless steel. Fig. 20 shows photographs of both incident faces and Fig. 21 shows the respective results. Both samples were Type A with a clearance of $d=0.52 \mathrm{~mm}$.

It is clear from the photographs in Fig. 20 that even for samples that were prepared in the same jig, the clearance with paper is less uniform than that for stainless steel. From Fig. 21, we infer that the absorption coefficient of the sample with paper was higher than the absorption coefficient of the sample with stainless steel. The maximum difference was about 0.1 . Hence, we predict that the absorption coefficient will change due to the nonuniformity of the clearance.

Next, we simulated the changes in the absorption coefficient due to the nonuniformity of the clearance through theoretical analysis. For Type A with clearance thickness of $0.52 \mathrm{~mm}$, Fig. 22 shows a schematic diagram of the case where changes in the clearance thickness occur due to the flexure and offset of the thin sheet. The flexure was assumed to have an arc shape with maximal radius at the middle of length $l$ in the $x$-direction. The offset is the amount by which the thin sheet shifts with respect to the clearance thickness. In this model, because the space between adjacent clearances is shared, the adjacent clearances are treated as a single set, as in Fig. 22 in the analysis. 

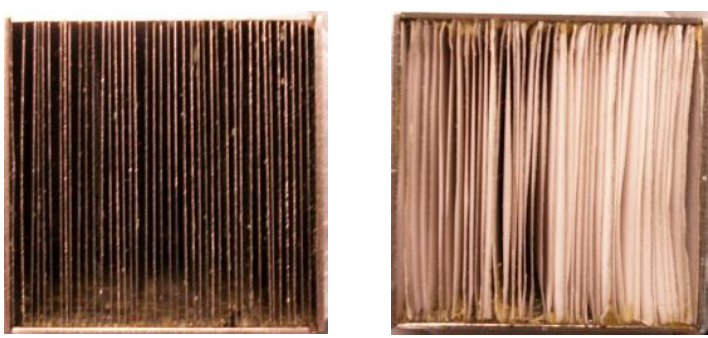

Fig. 20 Photograph of test sample made of stainless steel and paper (Type A, $d=0.52 \mathrm{~mm}$, thickness of sheet $0.08 \mathrm{~mm}$ )

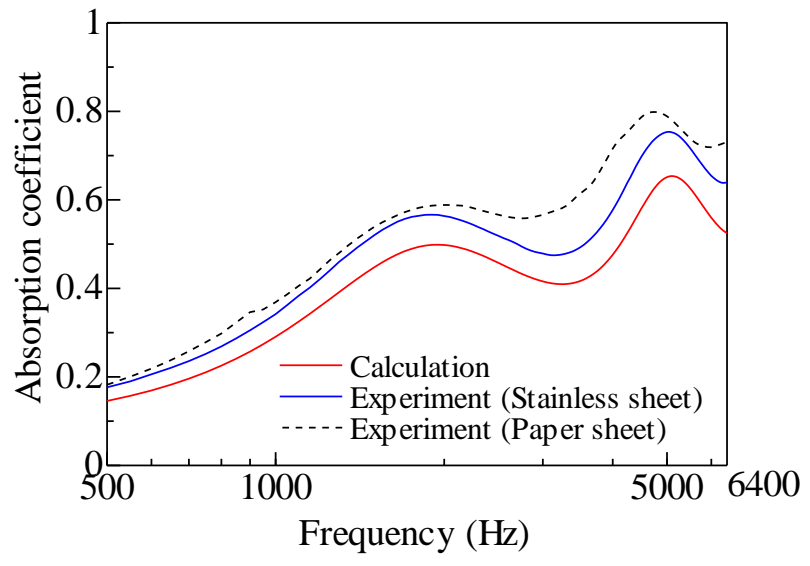

Fig. 21 Comparison between stainless steel and paper (Type A, $d=0.52 \mathrm{~mm}$ )

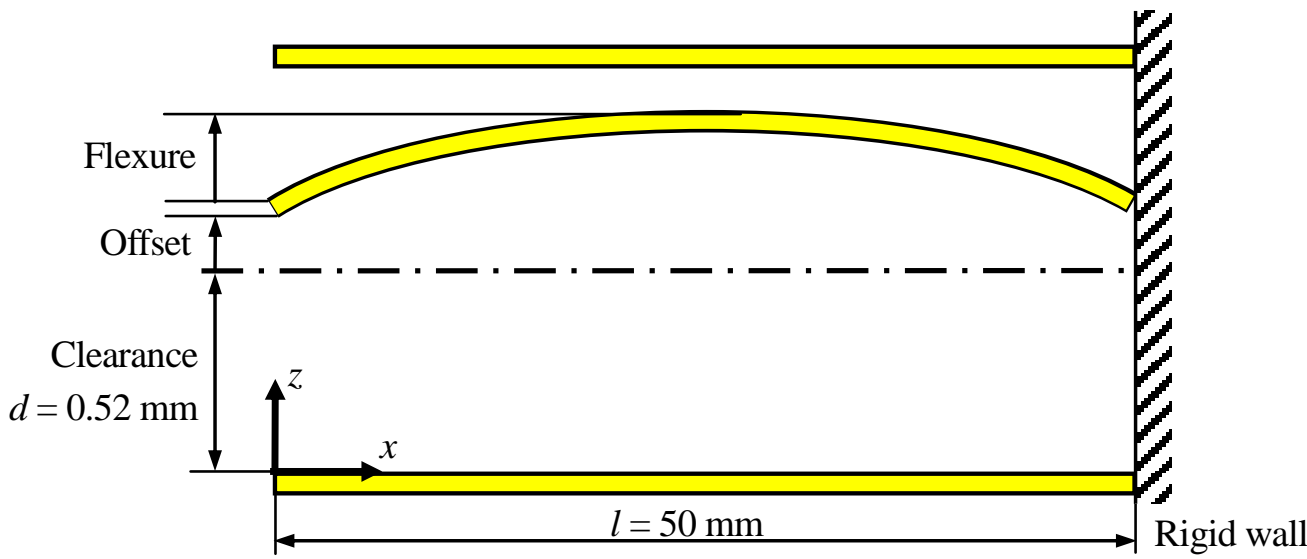

Fig. 22 Model of nonuniform clearances by flexure and offset of

Similarly to Type C, in the analysis for each clearance, the continuous variation in the clearance thickness in the $x$-direction is modeled by dividing the clearance in the $x$-direction and cascading the resulting transfer matrices. Furthermore, as shown in Fig. 22, the transfer matrices for the two differently shaped clearances were similarly connected in parallel as in Type B. For samples in which the total number of clearances is odd, the remaining clearance was assumed to have no flexure or offset.

We present the results of comparison of calculation results where there is only flexure (Fig. 23), offset (Fig. 24), and both flexure and offset (Fig. 25). The size of flexure and offset were given as percentages of the clearance thickness.

As shown in Figs. 23 and 24, the absorption coefficient changes only slightly when flexure or offset is 20 or $40 \%$. For $80 \%$ flexure, the absorption coefficient increases and the frequency range over which the calculated values are higher than the experiment values also increases. In contrast, for $80 \%$ offset, the absorption coefficient increases at high frequencies, whereas at low frequencies it is lower than when there is no flexure or offset. The calculated values for any offset are lower than the experimental values over the entire frequency range.

Next, in Fig. 25, we show the case where there are both flexure and offset on the thin sheet. When each of them is $20 \%$, the variation in the absorption coefficient is small and the calculated values approach the experimental ones. For $40 \%$ each, the calculated values are higher than the experimental values above the frequency of $1,350 \mathrm{~Hz}$.

Through theoretical analysis, we were able to simulate the causes of differences between the calculated and experimental values by considering the flexure and offset of the thin sheet. Moreover, in the calculated results in which either the flexure or the offset were changed, flexure contributed more to the changes in the absorption coefficient.

The calculated absorption coefficient for a thin sheet with offset in the opposite direction of that in Fig. 22 (i.e., $-40 \%$ ) was very close to the calculated absorption coefficient for $0 \%$ flexure or offset. This is likely because the offset of the thin sheet cancels out the effect of flexure. 


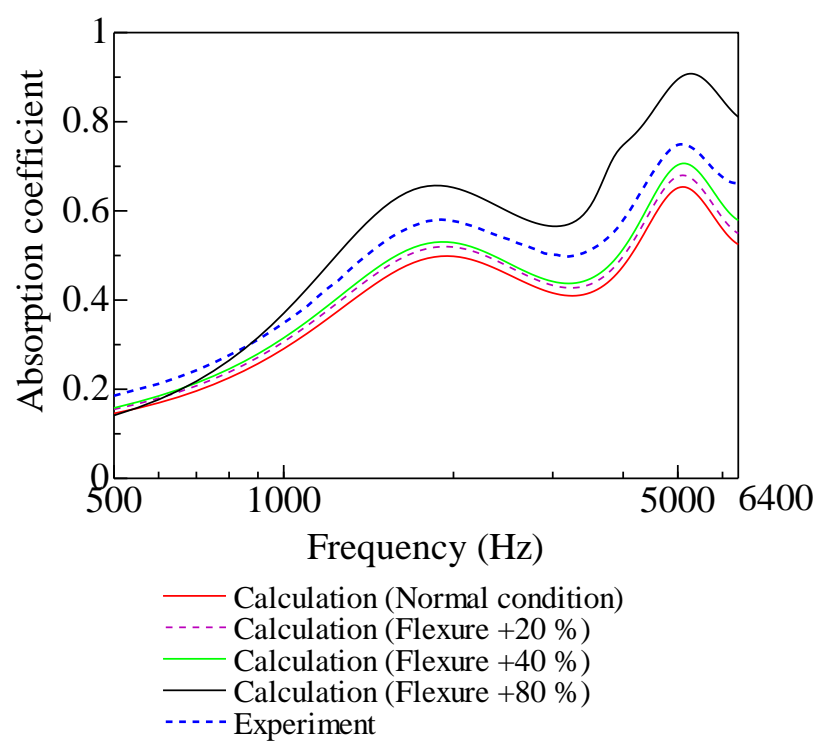

Fig. 23 Absorption coefficient of nonuniform clearances by flexure of sheets (Type A, $d=0.52 \mathrm{~mm}$ )

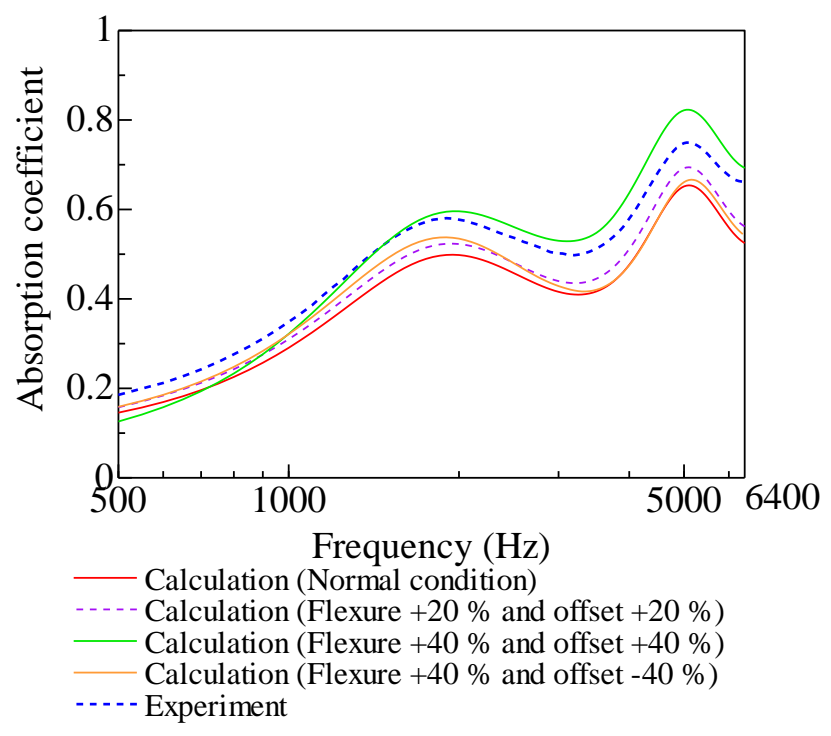

Fig. 25 Absorption coefficient of nonuniform clearances by flexure and offset of sheets

(Type A, $d=0.52 \mathrm{~mm}$ )

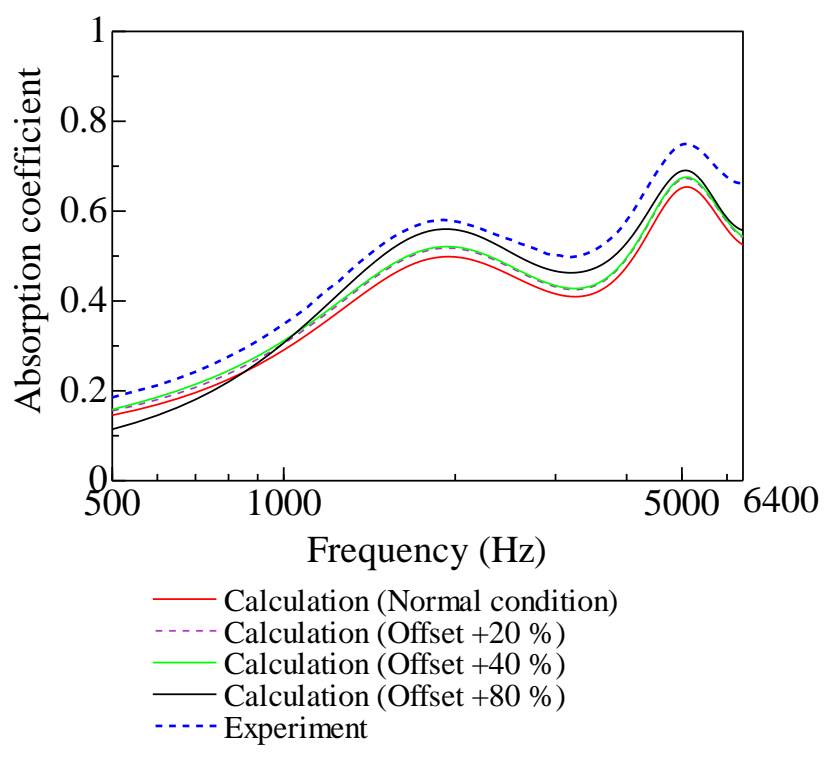

Fig. 24 Absorption coefficient of nonunifrom clearances by offset of sheets(Type A, $d=0.52 \mathrm{~mm}$ )

\section{Conclusion}

We carried out a theoretical analysis of sound-absorbing structures that uses the clearances between two planes formed with layered thin sheets by taking into account the viscosity of the boundary layer at the clearance. We also calculated the absorption coefficients for these structures. These calculation methods are useful in identifying the absorption coefficients for similar shapes and in designing sound-absorbing materials that use such shapes.

First, we examined the case of thin sheets placed in parallel.

Then, we examined the case where the size of the clearance continuously changed perpendicular to the direction of incidence of the sound waves. In this case, the transfer matrix used in the calculations was divided into elements perpendicular to the direction of incidence of the sound waves and the size of the clearance for each element was changed in a stepwise fashion.

Subsequently, we examined the case where the clearance continuously decreased in the direction of incidence of the 
sound waves. The transfer matrix used in the calculations is divided into elements in the direction of the incident sound waves and the size of the clearance changes in a stepwise fashion. In this case, the effect of the viscosity owing to the boundary layer differs depending on the frequency and the depth of the sample. Consequently, we obtained the effect like in case of the sound-absorbing material changes depending on the position in the depth direction of the sample. The sound absorption on both sides, and the transmission loss, can be simultaneously addressed by arranging incident face alternately; thus, these types of structures might be applied to sound-absorbing partitions.

The trends in the calculated data and the corresponding experimental data closely agreed. Two causes for the differences between the calculated and measured values were deduced. These are the nonuniformity in the thickness of the clearance and the flexure of the thin sheet material. As a theoretical analysis, the simulations were carried out by varying these two parameters. It was found that the differences between the calculated and measured values through simulations were comparatively less. In addition, the flexure of the thin sheet material was the main cause for the changes in the absorption coefficient.

As a result, it is reasonable to expect that waste paper, for example, could be reused in sound-absorbing structures with thin-walled materials. Reusing waste paper does not require energy to recycle and thus may help carbon fixation efforts.

\section{Nomenclature}

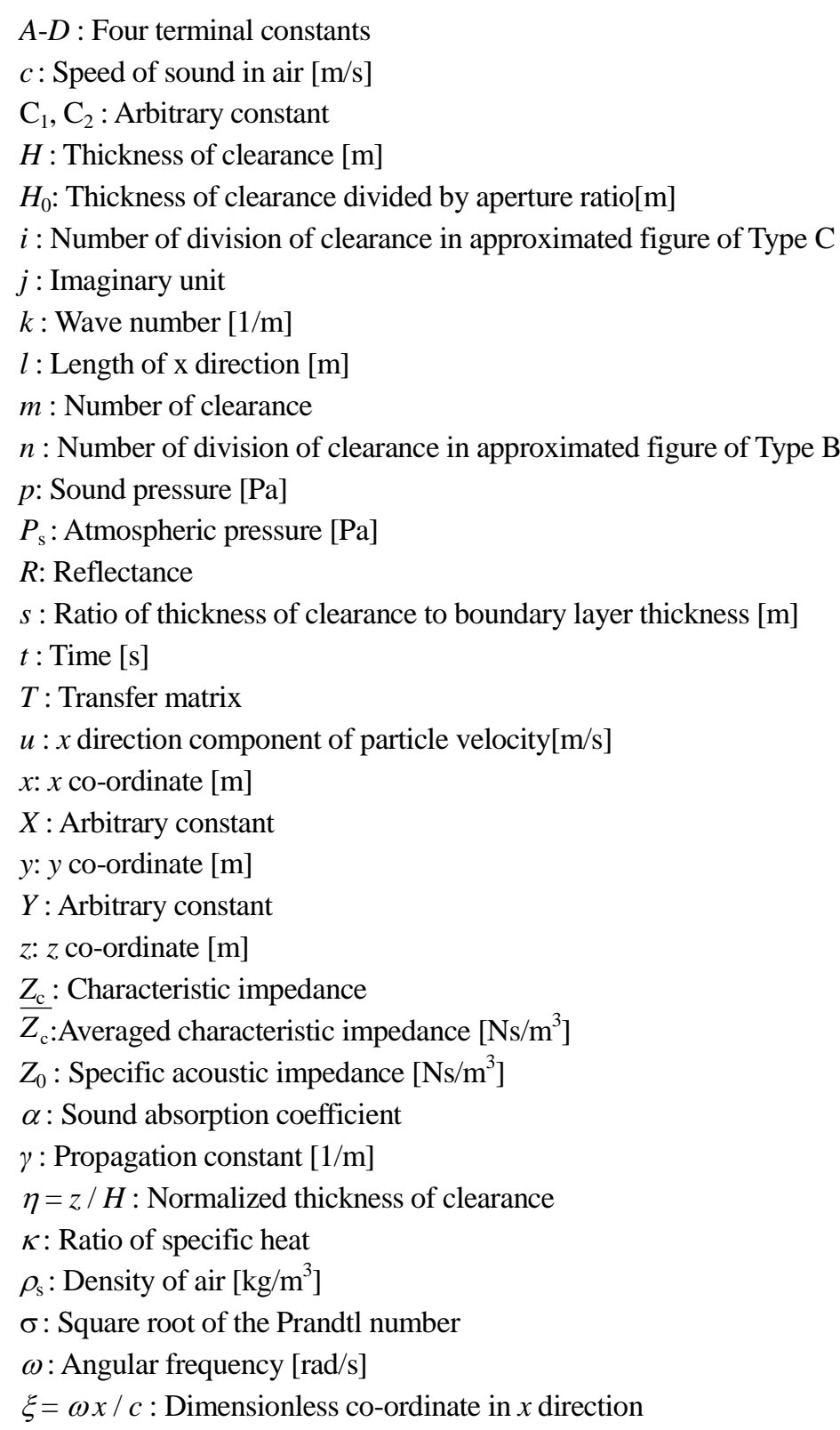




\section{Acknowledgment}

This work was supported by JSPS KAKENHI Grant Number 24560253.

\section{References}

Allard, J. F., Atalla, N., Sound Propagation in Porous Media: Modeling Sound Absorbing Materials, Second Edition, pp. 45-53, Wiley (2009).

Beltman, W. M., van der Hoogt, P. J. M., Spiering, R. M. E. J., Tijdeman, H., Implementation and Experimental Validation of New Viscothermal Acoustic Finite Element for Acousto-elastic Problems, Journal of Sound and Vibration, Vol. 216, No. 1 (1998), pp. 159-185.

Hirama, A., Kanda, K., Yokota, H., Hasagawa, N., Takada, S., Kato, K., Sound Absorption Characteristics of the Low Frequency Resonators that Utilized Recycled Paper, Bulletin of Tokyo Metropolitan Industrial Technology Research Institute, No. 6 (2003), pp. 37-40 (in Japanese).

Koshiroi, T., Tateishi, S., Features of Sound Absorption Data in Porous Material Caused by Elastic Frame Structure, Journal of Acoustical Society of Japan, Vol. 68, No. 9 (2012), pp. 474-479 (in Japanese).

McGinnes, C., Kleiner, M., Xiang, N., An Environmental and Economical Solution to Sound Absorption Using Straw, Journal of the Acoustical Society of America, Vol. 118, No. 3 (2005), pp. 1869-1869.

Nyborg, L. W., Acoustic Streaming near a Boundary, Journal of the Acoustical Society of America. Vol. 30, No. 4 (1958), pp. 329-339.

Sakamoto, S., Sugahara, R., Nagumo, T., Nakano, A., Kawase, H., Theoretical Analysis and Measurement of Sound Transmission Loss in Louver Elements with a Sound Attenuating Function Using a Helmholtz Resonator Array, Journal of Advanced Mechanical Design Systems and Manufacturing, Vol. 8, No. 3 (2014), Paper No.14-00061, 16 pages.

Sakamoto, S., Saito, K., Murayama, N., Higuchi, K., Experimental Studies on the Sound Absorbing Materials by Using of the Bundle of Narrow Clearances between Two Planes, Transactions of the Japan society of Mechanical Engineers, Series C, Vol. 79, No. 807 (2013a), pp. 4141-4152 (in Japanese).

Sakamoto, S., Hoshino, A., Sutoh, K., Sato, T., Estimating Sound-Absorption Coefficient and Transmission Loss by the Dimensions of Bundle of Narrow Holes (Comparison between Theoretical Analysis and Experiments), Transactions of the Japan society of Mechanical Engineers, Series C, Vol. 79, No. 807 (2013b), pp. 4164-4176 (in Japanese).

Sakamoto, S., Takauchi, Y., Yanagimoto, K., Watanabe, S., Study for Sound Absorbing Materials of Biomass Tubule etc. (Measured Result for Rice Straw, Rice Husks, and Buckwheat Husks), Journal of Environment and Engineering, Vol. 6, No. 2 (2011), pp. 352-364.

Stinson, R. M., Champou, Y., Propagation of Sound and the Assignment of Shape Factors in Model Porous Materials Having Simple Pore Geometries, Journal of the Acoustical Society of America, Vol. 91, No. 2 (1992), pp. 685-695.

Suyama, E., Hirata, M., Attenuation Constant of Plane Wave in a Tube : Acoustic Characteristic Analysis of Silencing Systems Based on Assuming of Plane Wave Propagation with Frictional Dissipation Part 1, The Journal of the Acoustical Society of Japan, Vol. 35, No. 4 (1979a), pp. 152-164 (in Japanese).

Suyama, E., Hirata, M., The Four Terminal Matrices of Tube System Based on Assuming of Plane Wave Propagation with Frictional Dissipation : Acoustic Characteristic Analysis of Silencing Systems Based on Assuming of Plane Wave Propagation with Frictional Dissipation Part 2, The Journal of the Acoustical Society of Japan, Vol. 35, No. 4 (1979b), pp. 165-170 (in Japanese).

Tijdeman, H., On the Propagation of Sound Waves in Cylindrical Tubes, Journal of Sound and Vibration, Vol. 39, No. 1 (1975), pp. 1-33.

Tsuchida, Y., Kobayashi, M., Examination of Practical Sound Absorption Coefficient by Material and the Composition, Toda Technical Research Report, Vol. 31 (2005) (in Japanese).

Utsuno, H., Tanaka, T., Fujikawa, T., and Seybert, A. F., Transfer Function Method for Measuring Characteristic Impedance and Propagation Constant of Porous Materials, J. Acoust. Soc. Am. Vol. 86 (1989), pp. 637-643.

Verdière, K., Panneton, R., Elkoun, S., Dupont, T., Leclaire, P., Transfer Matrix Method Applied to the Parallel Assembly of Sound Absorbing Materials, J. Acoust. Soc. Am., Vol. 134 (2013), pp. 4648-4658. 